\title{
Mitochondria-derived reactive oxygen species drive GANT61- induced mesothelioma cell apoptosis
}

\author{
Chuan Bian Lim ${ }^{1}$, Cecilia M. Prêle ${ }^{1,2}$, Svetlana Baltic ${ }^{1}$, Peter G. Arthur ${ }^{3}$, \\ Jenette Creaney ${ }^{4}$, D. Neil Watkins ${ }^{5}$, Philip J. Thompson ${ }^{1}$, Steven E. Mutsaers ${ }^{1,2}$ \\ ${ }^{1}$ Lung Institute of Western Australia and Centre for Asthma, Allergy and Respiratory Research, School of Medicine and \\ Pharmacology, University of Western Australia, Harry Perkins Institute of Medical Research, Nedlands, WA, Australia \\ ${ }^{2}$ Centre for Cell Therapy and Regenerative Medicine, School of Medicine and Pharmacology, University of Western Australia \\ and Harry Perkins Institute of Medical Research, Nedlands, WA, Australia \\ ${ }^{3}$ School of Chemistry and Biochemistry, University of Western Australia, Crawley, WA, Australia \\ ${ }^{4}$ National Centre for Asbestos Related Diseases, School of Medicine and Pharmacology, University of Western Australia, Harry \\ Perkins Institute of Medical Research and Australian Mesothelioma Tissue Bank, Sir Charles Gairdner Hospital, Nedlands, \\ WA, Australia \\ ${ }^{5}$ Garvan Institute of Medical Research, Darlinghurst, NSW, Australia
}

Correspondence to:

Steven E. Mutsaers, e-mail: mutsaers@liwa.uwa.edu.au

Keywords: Apoptosis, mesothelioma, GANT61, Hedgehog, reactive oxygen species

Received: September 02, $2014 \quad$ Accepted: November 08, $2014 \quad$ Published: January 16, 2015

\section{ABSTRACT}

Gli transcription factors of the Hedgehog ( $\mathrm{Hh}$ ) pathway have been reported to be drivers of malignant mesothelioma (MMe) cell survival. The Gli inhibitor GANT61 induces apoptosis in various cancer cell models, and has been associated directly with Gli inhibition. However various chemotherapeutics can induce cell death through generation of reactive oxygen species (ROS) but whether ROS mediates GANT61-induced apoptosis is unknown. In this study human MMe cells were treated with GANT61 and the mechanisms regulating cell death investigated. Exposure of MMe cells to GANT61 led to G1 phase arrest and apoptosis, which involved ROS but not its purported targets, GLI1 or GLI2. GANT61 triggered ROS generation and quenching of ROS protected MMe cells from GANT61-induced apoptosis. Furthermore, we demonstrated that mitochondria are important in mediating GANT61 effects: (1) ROS production and apoptosis were blocked by mitochondrial inhibitor rotenone; (2) GANT61 promoted superoxide formation in mitochondria; and (3) mitochondrial DNA-deficient L068 cells failed to induce superoxide, and were more resistant to apoptosis induced by GANT61 than wild-type cells. Our data demonstrate for the first time that GANT61 induces apoptosis by promoting mitochondrial superoxide generation independent of Gli inhibition, and highlights the therapeutic potential of mitochondrial ROS-mediated anticancer drugs in MMe.

\section{INTRODUCTION}

Malignant mesothelioma (MMe) is an aggressive fatal cancer, predominantly of the pleura and peritoneum, primarily caused by occupational asbestos exposure [1]. Current approaches involving surgery, radiotherapy or chemotherapy have failed to bring a clear survival benefit to patients, with a median survival of less than 12 months from diagnosis [2]. Clearly, new therapeutic strategies are urgently needed to improve the survival rate and quality of life of these patients.
The Hedgehog (Hh) pathway is a conserved signaling pathway responsible for the regulation of pattern specification during embryonic development [3]. It is also involved in the control of adult tissue homeostasis and stem cell maintenance [4]. For Hh signaling, the Hh protein acts as a ligand for the Patched1 (PTCH1) transmembrane receptor proteins [5]. PTCH1 interaction with transmembrane signal transducer Smoothened (SMO) under normal conditions inhibits SMO function [6]. Upon binding of Hh ligand to PTCH1, the inhibition of SMO by PTCH1 is alleviated, resulting in the activation of Gli 
transcription factors, capable of regulating expression of Hh target genes [7].

Several small molecule compounds with inhibitory effects on the Hh pathway have been reported. Notably, US FDA has approved GDC-0449, a SMO antagonist, for the treatment of patients with advanced stages of basal cell carcinoma [8]. Besides targeting SMO, the Hh ligand and Gli transcription factors appear highly amenable to inhibition by small molecule inhibitors. Of particular interest is GANT61, discovered in a cell-based screen for antagonists of Gli-mediated transcription [9]. GANT61 induces apoptosis in various human cancer cells including MMe [10-13] and suppresses the growth of prostate carcinoma [9] and neuroblastoma [14] xenografts in mice, suggesting that Gli proteins could be a therapeutic target in these cancers. The cytotoxic effect of GANT61 was previously attributed to the inhibition of Gli binding to DNA [9], thus preventing the transcription of Hh target genes such as Bcl-2 [10, 15], which are involved in cell proliferation and apoptosis. Recent studies showed that GANT61 induced autophagic death in hepatocellular carcinoma (HCC) cells through upregulation of Bnip3 [16]. However, it is unclear whether GANT61 have effects independent of $\mathrm{Hh} / \mathrm{Gli}$ signaling.

Reactive oxygen species (ROS) are traditionally viewed as toxic molecules that damage cellular DNA, proteins and lipids [17]. However recently, ROS have been shown to have important physiological signaling functions [18]. Generation of ROS in response to chemo- and radio-therapy has been reported to induce cell cycle arrest and/or cell death in various cancer models [19]. However, whether GANT61 induces ROS generation has not been reported. Therefore, we examined the role of ROS in mediating GANT61induced apoptosis and determined if this occurred via Hh/Gli signaling.

\section{RESULTS}

\section{GANT61 induces G1 cell cycle arrest and apoptosis in human MMe cells}

The antiproliferative activity of GANT61 was assessed in MMe cells. GANT61 inhibited cell proliferation in all MMe cell lines in a dose-dependent manner (Figure 1A). Among the cell lines tested, JU77 cells showed highest sensitivity to GANT61 treatment $\left(\mathrm{IC}_{50}=4.02 \mu \mathrm{M}\right)$ whereas NO36 cells appeared to be the most resistant $\left(\mathrm{IC}_{50}=31.8\right.$ $\mu \mathrm{M})$ (Figure 1A). As shown in Figure 1B, concomitant with growth inhibitory effect, GANT61 induced G1 cell cycle arrest as indicated by the increased percentage of LO68 cells in G1 cell cycle phase at $24 \mathrm{~h}$ compared to vehicletreated cells. There was also an accumulation of cells in the sub-G1 fraction at 48-72 h compared to vehicle-treated cells, suggesting that apoptosis is involved (Figure 1C). To understand the cell death response to GANT61 in LO68 cells, cells were treated with GANT61 and phosphatidylserine externalization, a marker of early apoptosis, determined.
A

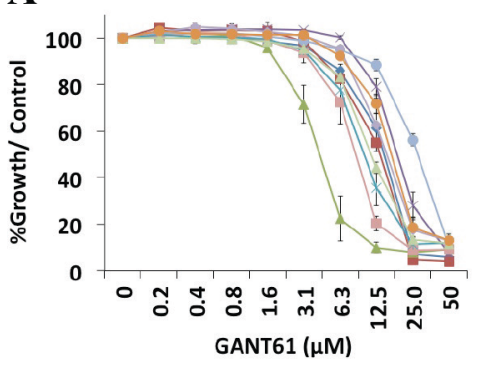

C

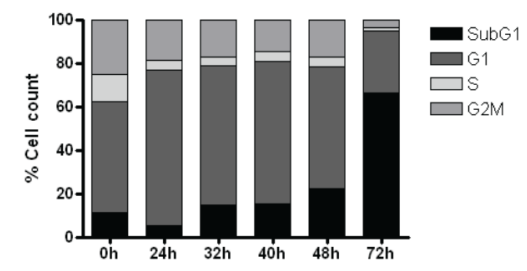

B
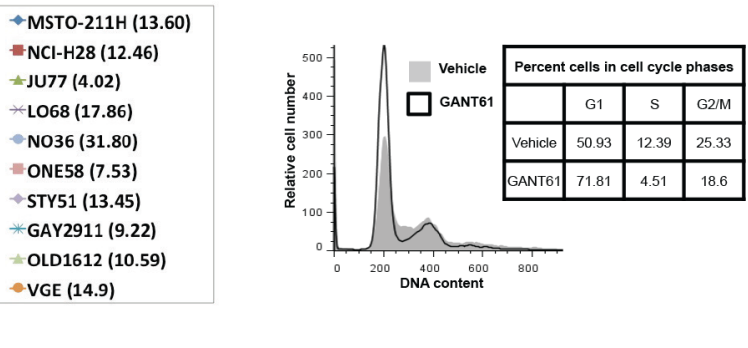

D

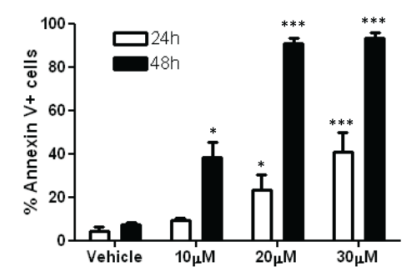

Figure 1: GANT61 induces G1 phase arrest and apoptosis in human MMe cells. (A) Dose-response cytotoxicity curves for MMe cells treated with GANT61 for $72 \mathrm{~h}$. The dose range tested was $0-50 \mu \mathrm{M}$. $\mathrm{IC}_{50}$ values are shown in brackets for each cell line. Values are the mean of independent experiments performed in 6 replicates (mean \pm SEM; $n=3$ ). (B) Cell cycle analysis of LO68 cells treated for $24 \mathrm{~h}$ with either $20 \mu \mathrm{M}$ GANT61 (open graph) or vehicle (grey graph). The inset shows the percentage of cells at different phases of the cell cycle (G1, S and G2/M) of GANT61- and vehicle-treated cells. (C) Cell cycle analysis of LO68 cells treated for 0-72 h with $20 \mu \mathrm{M}$ GANT61. (D) Apoptosis (as assessed by the annexin V/7AAD assay) was quantified in LO68 cells treated with 10, 20 or $30 \mu \mathrm{M}$ GANT61 or vehicle for $24-48 \mathrm{~h}$. Bar graphs show the quantification of results from independent experiments (mean $\pm \mathrm{SEM}, n=3$ ). *, $p<0.05$ or ***, $p<0.001$, compared to vehicle-treated cells. 
GANT61 induced a parallel increase in apoptotic cell death in a dose- and time-dependent manner, as indicated by FACS analysis of annexin V-binding (Figure 1D).

\section{GANT61 targets Gli transcription factors in MMe cells}

GANT61 reduced mRNA expression of GLI1 and GLI2 following treatment with $20 \mu \mathrm{M}$ GANT61 for up to $72 \mathrm{~h}$ (Figure 2A) as well as the Gli downstream target gene PTCH1 (Figure 2A). A similar downregulation of GLI1 and GLI2 proteins was observed after 24 $\mathrm{h}$ exposure to different concentrations of GANT61 $(10-30 \mu \mathrm{M})$ (Figure 2B). The protein level of Bcl-2, a GLI1 downstream target gene [20], was also reduced after GANT61 treatment (Figure 2B). To confirm the specificity of inhibition of GLI1 and GLI2 by GANT61, we tested its efficacy in a Gli luciferase reporter assay.
Consistent with previous findings, GANT61 inhibited the Gli reporter activity in LO68 cells (Figure 2C). These findings point to GANT61 being an inhibitor of GLI1 and GLI2 [9].

\section{GANT61 induces oxidative stress}

Previous studies showed that GANT61 can induce DNA damage in colon cancer cells [10]. We hypothesize that GANT61 triggers the production of reactive oxygen species (ROS), which in turn damages DNA. To test this hypothesis, cells were treated with GANT61 $(10-20 \mu \mathrm{M})$ for 24 to $48 \mathrm{~h}$ and intracellular ROS levels were measured using the carboxy derivative of fluorescein, $\mathrm{CH}_{2} \mathrm{DCFDA}$. As shown in Figure 3A, ROS levels increased significantly in LO68 cells treated with GANT61 in a dose- and time-dependent manner. GANT61 also triggered ROS generation in HCT116 and HT29 colon cancer cells,

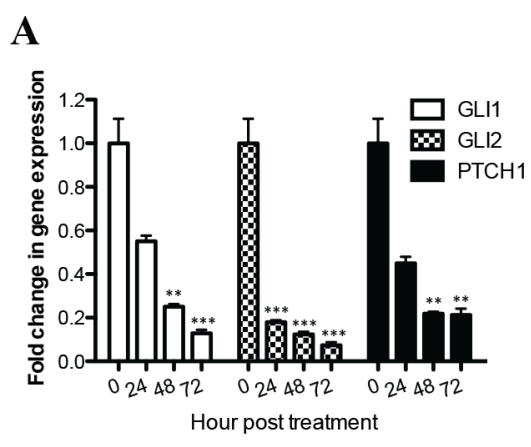

B

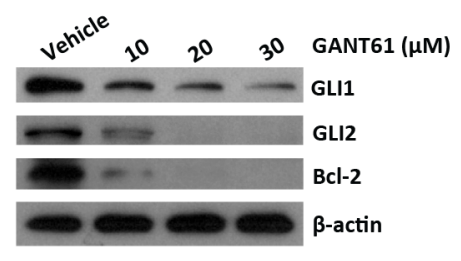

C

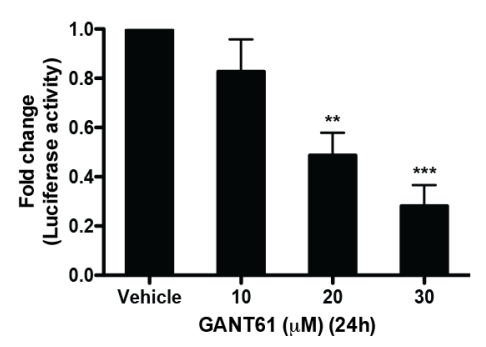

Figure 2: GANT61 targets Gli transcription factors in MMe cells. (A) qRT-PCR analysis of the Hh pathway genes GLII, GLI2 and PTCH1 was performed on LO68 cells treated with $20 \mu \mathrm{M}$ GANT61 or vehicle for 24-72 $\mathrm{h}$. The expression levels of each gene were normalized using PGK1 mRNA as an endogenous control and are indicated as the fold change with respect to the vehicle-treated LO68 cells. Values represent the mean \pm SEM of three independent experiments each performed in duplicate. ${ }^{* *}, p<0.01$ or $* * *, p<0.001$, compared to vehicle-treated cells. (B) Western blot analysis of GLI1, GLI2, Bcl-2 and $\beta$-actin on LO68 cells treated with 10, 20 or 30 $\mu$ M GANT61 or vehicle for 24 h. (C) Gli transcriptional activity was determined by transfecting LO68 cells with a Gli-responsive luciferase reporter plasmid. Cells were treated with either 10, 20 or $30 \mu \mathrm{M}$ GANT61 or vehicle for $24 \mathrm{~h}$. Luciferase activity of cell lysates was measured and normalized to Renilla luciferase activity obtained by co-transfection with a constitutively expressed Renilla luciferase internal control plasmid. Results are expressed as the mean \pm SEM from three independent experiments. ${ }^{* *}, p<0.01$ or ${ }^{* * *}, p<0.001$, compared to vehicle-treated cells. 
suggesting that the production of ROS could be a general effect of GANT61 exposure (Figure 3B). Moreover, pretreatment of LO68 cells with $\mathrm{N}$-acetylcysteine (NAC) and reduced L-glutathione (GSH), two potent ROS scavengers, attenuated this accumulation of ROS (Figure 3C). As shown in Figure 3D, neutralization of ROS by NAC in GANT61-treated cells restored cell viability, suggesting that ROS is responsible for GANT61 cytotoxicity. Consistent with this data, annexin V/7AAD assays showed that NAC pretreatment rescued LO68 cells from GANT61-induced apoptosis (Figure 3E).

\section{GANT61 downregulates GLI1, GLI2 and PTCH1 through ROS}

We next examined the effect of NAC on GANT61mediated GLI1, GLI2 and PTCH1 expression. As shown in Figure 4A, the downregulation of GLI1, GLI2 and PTCH1 expression by GANT61, as determined by qRT-PCR, was abolished by pretreating cells with NAC. The blockade of ROS accumulation by NAC prevents reduction of GLII, GLI2 and PTCH1 expression indicating the involvement of ROS in modulation of the Hh pathway. Stimulated by our novel finding that ROS could potentially impact on

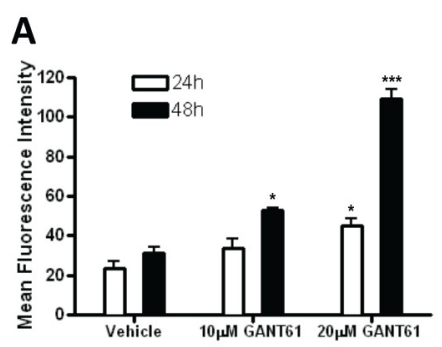

B
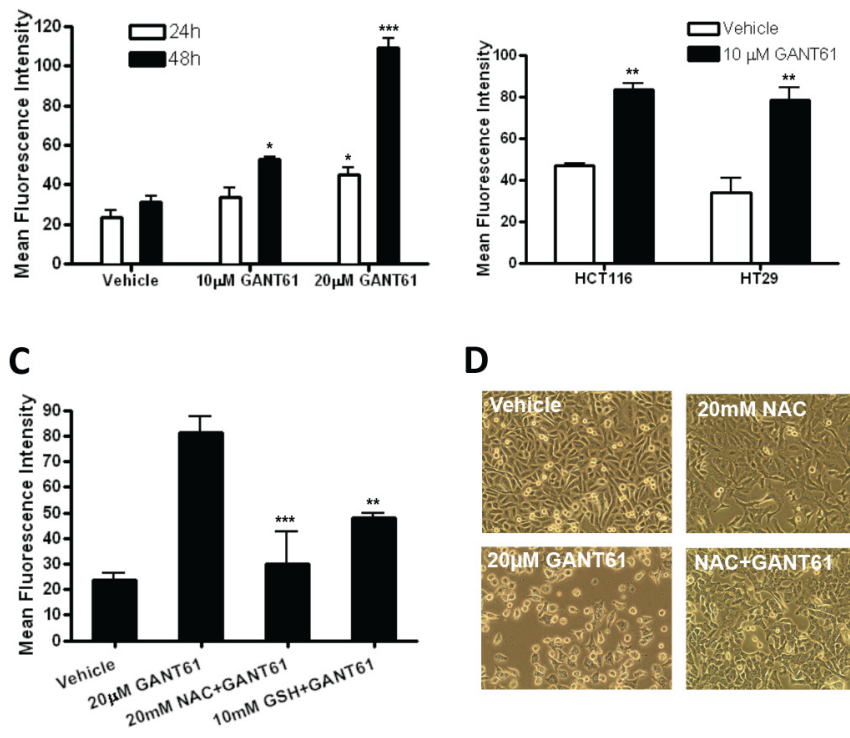

D
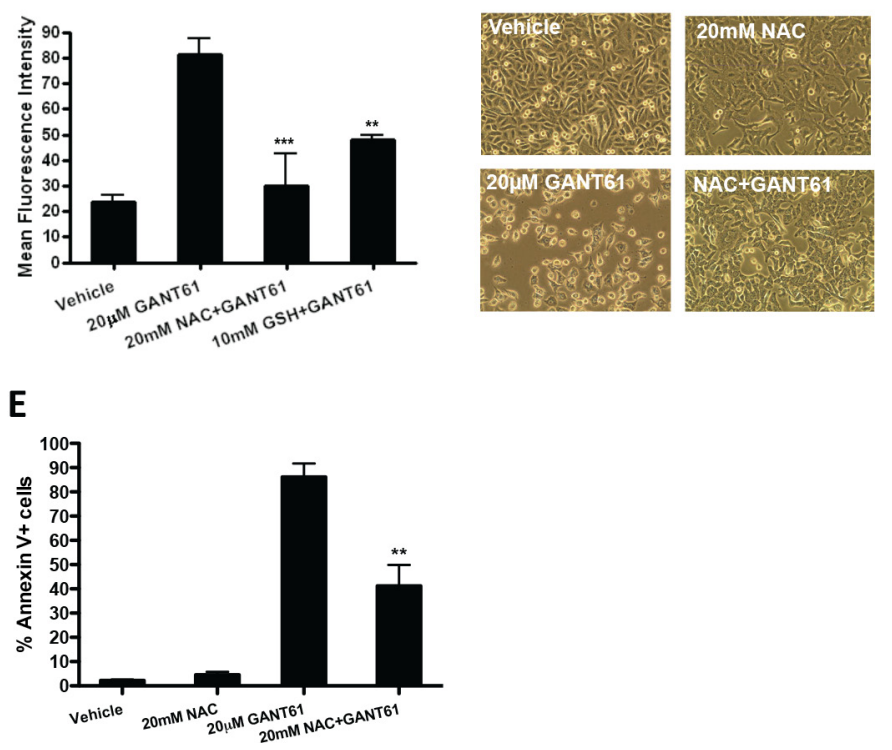

Figure 3: Oxidative stress is involved in GANT61-induced apoptosis. (A) GANT61 treatment in LO68 cells is associated with an increase in ROS in a dose-and time-dependent manner. LO68 cells were treated with 10 and $20 \mu \mathrm{M}$ GANT61 or vehicle for 24 or 48 h. The cells were stained with the fluorescent probe $\mathrm{CH}_{2}$ DCFDA, and the fluorescence was measured by flow cytometry. Data are expressed as mean fluorescence intensity of positive cells measured in each experimental condition. Values are the average of independent measurements (mean $\pm \mathrm{SEM} ; n=3$ ). ${ }^{*}, p<0.05$ or ***, $p<0.001$, compared to vehicle-treated cells. (B) ROS is induced in GANT61-treated colon cancer

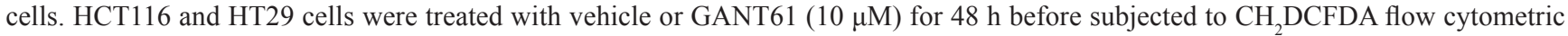
analysis. Values are the average of independent measurements (mean $\pm \mathrm{SEM} ; n=3$ ). ${ }^{* *}, p<0.01$, compared to vehicle-treated cells. (C) LO68 cells were treated with vehicle or $20 \mu \mathrm{M}$ GANT61 for $48 \mathrm{~h}$ with or without the antioxidants NAC (20 mM) and GSH (10 mM). Bar graph shows the increase in the mean fluorescence intensity of $\mathrm{CH}_{2}$ DCFDA-positive cells measured in each experimental condition. Values are the average of independent measurements (mean $\pm \mathrm{SEM} ; n=3$ ). **, $p<0.01$ or ***, $p<0.001$, compared to vehicle-treated cells. (D) Representative light micrographs showing the effects of $20 \mu \mathrm{M}$ GANT61 or vehicle after $48 \mathrm{~h}$ with or without $20 \mathrm{mM}$ antioxidant NAC on the morphology of LO68 cells. Note the reduced cell growth and altered cell morphology in GANT61-treated LO68 cells, which were not seen in vehicle-treated cells. Pretreatment with the antioxidant NAC restores cell growth and abrogates apoptosis in LO68 cells treated with $20 \mu \mathrm{M}$ GANT61 for $24 \mathrm{~h}$. (E) Apoptosis (as assessed by the annexin V/7AAD assay) was quantified in LO68 cells treated with vehicle or GANT61 $(20 \mu \mathrm{M})$ with or without $20 \mathrm{mM}$ antioxidant NAC for $48 \mathrm{~h}$. Bar graphs show results from independent experiments (mean \pm SEM, $n=3$ ). ${ }^{* *}, p<0.01$, compared to GANT61-treated cells. 
the Hh pathway, we next assessed the effect of exposing LO68 cells to menadione, a ROS generator, and hydrogen peroxide $\left(\mathrm{H}_{2} \mathrm{O}_{2}\right)$, a mimic of oxidative stress, on the $\mathrm{Hh}$ pathway. FACS analysis of intracellular ROS production indicated that exposure of LO68 cells to menadione and $\mathrm{H}_{2} \mathrm{O}_{2}$ resulted in a significant increase in ROS production as measured by the fluorescent $\mathrm{CH}_{2}$ DCFDA probe (Figure $4 \mathrm{~B}$ and 4C). Furthermore, qRT-PCR analysis of gene expression in LO68 cells following treatment clearly indicated the ability of menadione and $\mathrm{H}_{2} \mathrm{O}_{2}$ to downregulate the expression of GLI1, a marker of $\mathrm{Hh}$ pathway activity (Figure 4D and 4E). Together, these findings suggest that ROS plays a critical role in the suppression of GLI1, GLI2 and PTCH1 expression by GANT61.

\section{GANT61-induced ROS, apoptosis and cell cycle arrest are independent of Gli inhibition}

Because GANT61 is an inhibitor of Gli transcription factors, we set out to determine whether GANT61-induced G1 cell cycle arrest, apoptosis and ROS production are dependent on Gli inhibition. First, we examined apoptosis and ROS production in LO68 cells following siRNAmediated depletion of GLI1 and GLI2. qRT-PCR analyses demonstrated knockdowns of GLI1 and GLI2 mRNA in LO68 cells (Figure 5A). Surprisingly however, neither individual GLI1 or GLI2 knockdowns nor their combined depletion induced cell death as indicated by the small sub-G1 apoptotic population $(<5 \%$ in sub-G1 phase in GLI1- and/or GLI2-depleted cells compared to $\sim 5 \%$ cells

\section{B}
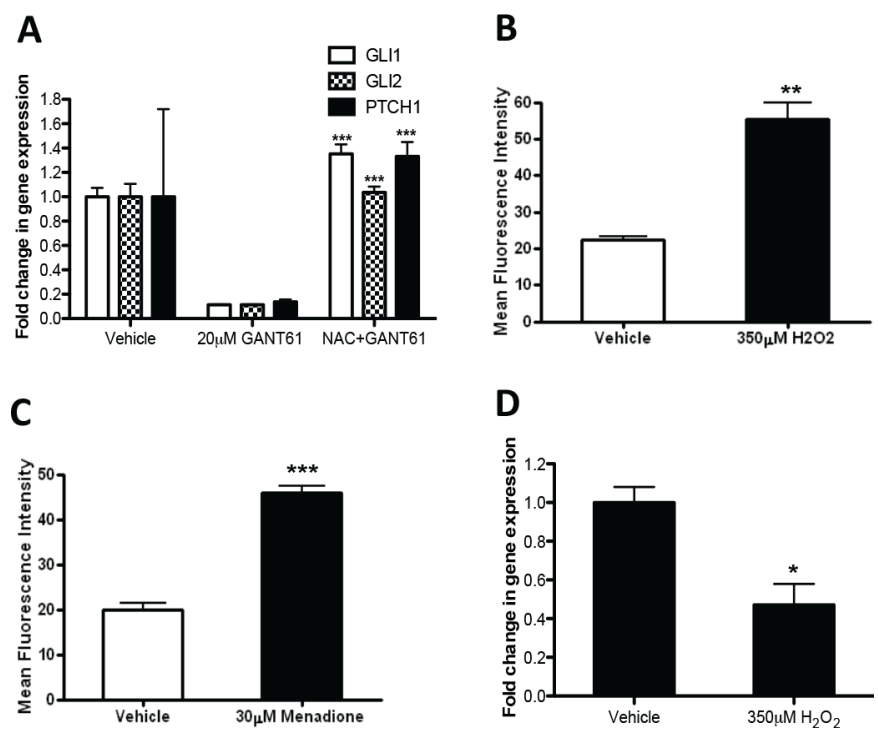

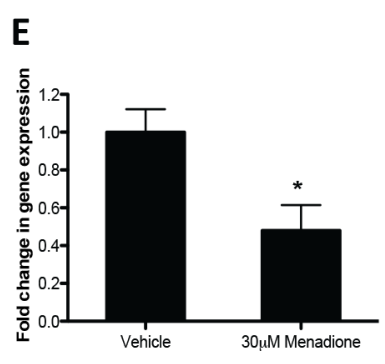

Figure 4: GANT61 downregulates GLI1, GLI2 and PTCH1 through ROS. (A) Analyses of Hh pathway genes (GLII, GLI2 and $P$ TCH1) by qRT-PCR. LO68 cells were pretreated with NAC for $1 \mathrm{~h}$ and then with $20 \mu \mathrm{M}$ GANT61 or vehicle for $48 \mathrm{~h}$. Values represent the mean \pm SEM of three independent experiments each performed in duplicate. ${ }^{* * *}, p<0.001$, compared to GANT61-treated cells. (B) LO68 cells were treated with vehicle or $350 \mu \mathrm{M} \mathrm{H}_{2} \mathrm{O}_{2}$ for $24 \mathrm{~h}$. The cells were stained with $\mathrm{CH}_{2}$ DCFDA and the fluorescence measured by flow cytometry. Bar graph represents the increase in mean fluorescence intensity of positive cells measured in each experimental condition. Values are the average of independent measurements (mean $\pm \mathrm{SEM} ; n=3$ ). ${ }^{* *}, p<0.01$, compared to vehicle-treated cells. (C) LO68 cells were treated with vehicle or $30 \mu \mathrm{M}$ menadione for $24 \mathrm{~h}$. The cells were stained with $\mathrm{CH}_{2}$ DCFDA and fluorescence measured by flow cytometry. Bar graph shows the increase in the mean fluorescence intensity of positive cells measured in each experimental condition. Values are the average of independent measurements (mean $\pm \mathrm{SEM} ; n=3$ ). ${ }^{* * *}, p<0.001$, compared to vehicle-treated cells. (D) Analysis of GLII mRNA expression by qRT-PCR. LO68 cells were treated with vehicle or $350 \mu \mathrm{M} \mathrm{H}_{2} \mathrm{O}_{2}$ for $24 \mathrm{~h}$. Values represent the mean \pm SEM of three independent experiments each performed in duplicates. ${ }^{*}, p<0.05$, compared to vehicle-treated cells. (E) Analysis of GLI1 mRNA expression by qRT-PCR. LO68 cells were treated with vehicle or $30 \mu \mathrm{M}$ menadione for $24 \mathrm{~h}$. Values represent the mean \pm SEM of three independent experiments each performed in duplicates. ${ }^{*}, p<0.05$, compared to vehicle-treated cells. 
in sub-G1 phase in LO68 cells transiently transfected with negative control (NC) siRNA), indicating that the proapoptotic activity of the drug is likely to be independent of Gli inhibition (Figure 5B). Similarly, the results from FACS analysis showed that the knockdowns of GLI1 and GLI2 by siRNA did not result in G1 cell cycle arrest ( $\sim 60 \%$ in G1 phase in GLI1- and/or GLI2-depleted cells compared to $\sim 60 \%$ cells in G1 phase in LO68 cells transiently transfected with NC siRNA) (Figure 5C). Moreover, depletion of GLI1 and GLI2, individually or together using siRNAs did not result in ROS generation in LO68 cells (Figure 5D). Taken together, our data suggest that generation of ROS and induction of apoptosis and cell cycle arrest in response to GANT61 is independent of Hh/Gli signaling in LO68 cells.

\section{GANT61-induced apoptosis and ROS production is dependent on mitochondria}

The mitochondrion is a major site of ROS generation in mammalian cells [18]. To determine the site of ROS production in response to GANT61, LO68 cells were treated with GANT61 in the absence or presence of rotenone, a mitochondrial complex I inhibitor, and the effects on GANT61-induced ROS generation and apoptosis were assessed. GANT61-induced ROS production (Figure 6A) and apoptosis (Figure 6B) were significantly blocked by the addition of rotenone, indicating that the ROS produced in response to GANT61 was of mitochondrial origin. The addition of a mitochondria-targeted antioxidant, MitoTEMPO, also blocked the increase in apoptosis induced by GANT61 (Figure 6C). We further confirm the mitochondrial origin of ROS by measuring the levels of superoxide within mitochondria after exposure to GANT61 using the fluorescent probe mitoSOX red. As shown in Figure 6D, exposure to $20 \mu \mathrm{M}$ GANT61 for $48 \mathrm{~h}$ induced an increase in intramitochondrial superoxide levels. In addition, this increase in intramitochondrial superoxide was attenuated by pretreating cells for $1 \mathrm{~h}$ with $20 \mathrm{mM}$ NAC before co-treating with $20 \mu \mathrm{M}$ GANT61 (Figure 6D).

\section{Mitochondrial superoxide is essential for GANT61-induced apoptosis}

To genetically confirm involvement of mitochondrial superoxide in GANT61-induced apoptosis, we generated LO68 $\rho^{0}$ cells, which lack mitochondrial DNA, by exposing cells to low concentration of ethidium bromide. Mitochondrial DNA depletion was verified in LO68 $\rho^{0}$ cells by amplifying three mitochondria-encoded genes, $C O X 1, D$-loop and ND6, by PCR. In addition, GADPH was amplified to serve as a control for nuclear-encoded genes. As
A

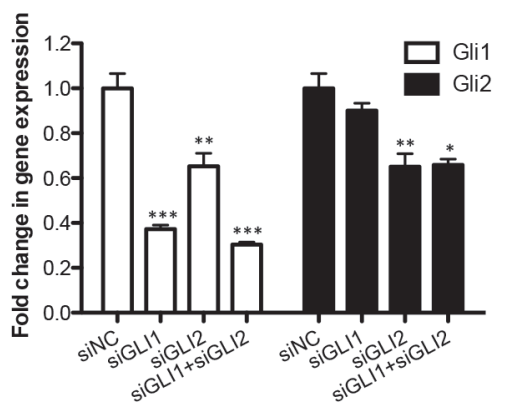

C

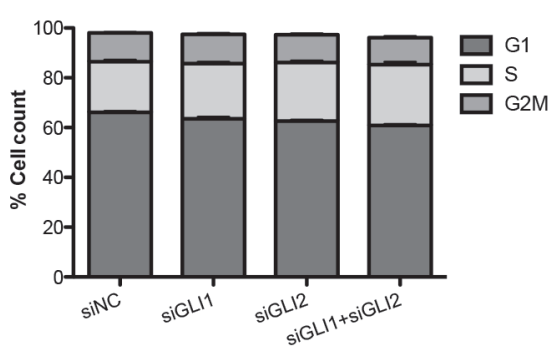

B

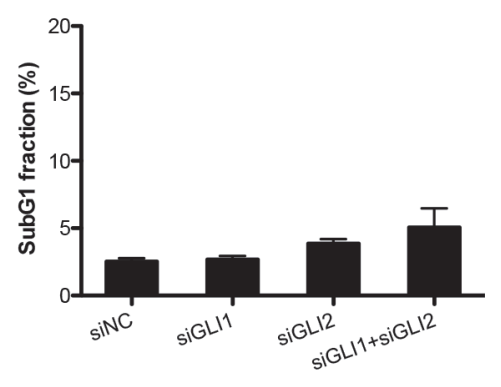

D

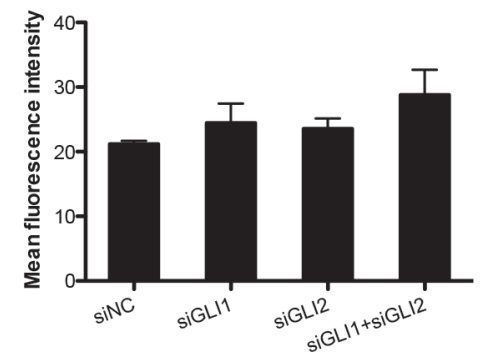

Figure 5: Knockdown of GLI1 and GLI2 by siRNA does not increase ROS production in MMe cells. (A) LO68 cells were transfected with negative control (siNC), GLI1 (siGLI1) or GLI2 (siGLI2) siRNA for $96 \mathrm{~h}$, then GLI1 mRNA expression analyzed by qRTPCR. Values represent the mean \pm SEM of three independent experiments each performed in duplicates. $*, p<0.05, * *, p<0.01$ or $* * *$, $p<0.001$, compared to NC siRNA-transfected cells. (B) Cell death (subG1 fraction) was measured by flow cytometry $96 \mathrm{~h}$ after transfection. Data represent the mean \pm SEM of three independent experiments. (C) The cell cycle was analyzed by flow cytometry $96 \mathrm{~h}$ following transfection. Histogram profiles of flow-cytometric analysis show the cell cycle distribution of the cell population. Data represent the mean \pm SEM of three independent experiments. (D) The level of intracellular ROS was monitored using $\mathrm{CH}_{2} \mathrm{DCFDA}_{\text {and }}$ the fluorescence measured by flow cytometry $96 \mathrm{~h}$ following transfection. Data represent the mean $\pm \mathrm{SEM}$ of three independent experiments. 
A

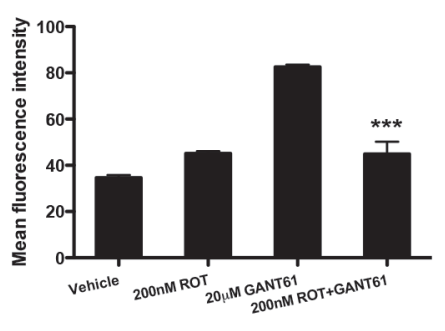

C

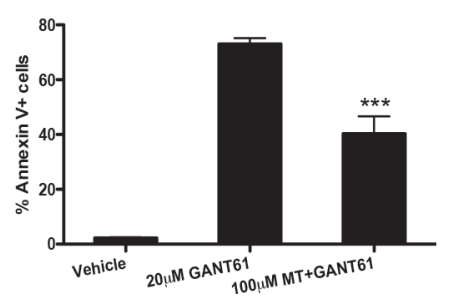

B

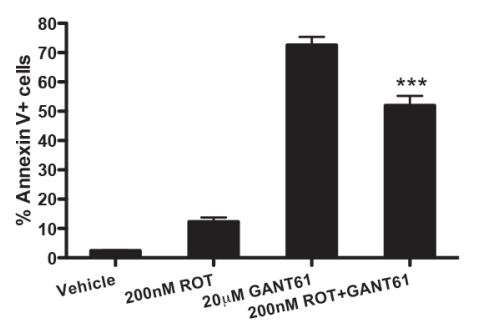

D

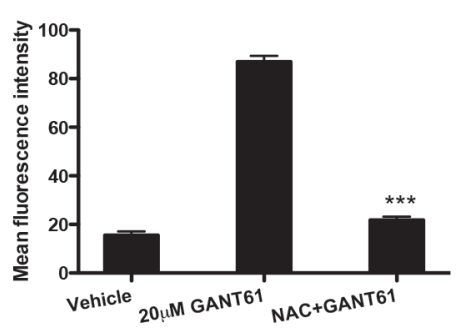

Figure 6: GANT61-induced apoptosis and ROS production is dependent on mitochondria. (A) LO68 cells were pretreated with $200 \mathrm{nM}$ rotenone (ROT), an inhibitor of mitochondrial complex 1, for $1 \mathrm{~h}$, and further incubated with $20 \mu \mathrm{M}$ GANT61 for $48 \mathrm{~h}$. The level of intracellular ROS was monitored using $\mathrm{CH}_{2}$ DCFDA and the fluorescence measured by flow cytometry. Data represent the mean \pm SEM of three independent experiments. ${ }^{* *}, p<0.001$, compared to GANT61-only treated cells. (B) Apoptosis was measured by annexin $\mathrm{V} / 7 \mathrm{AAD}$ assay. Data represent the mean \pm SEM of three independent experiments. ${ }^{* * *}, p<0.001$, compared to GANT61-only treated cells. (C) Mitochondria-targeted superoxide dismutase mimetic mitoTEMPO (MT) attenuates GANT61-induced apoptosis. LO68 cells were pretreated with MT $(100 \mu \mathrm{M})$ for $1 \mathrm{~h}$ and further incubated with $20 \mu \mathrm{M}$ GANT61 for $48 \mathrm{~h}$. Apoptosis was measured by annexin$\mathrm{V} / 7 \mathrm{AAD}$ assay. Data represent the mean \pm SEM of three independent experiments. ***, $p<0.001$, compared to GANT61-only treated cells. (D) GANT61 induces mitochondrial superoxide production in LO68 cells. Cells were pretreated with NAC (20 mM) for $1 \mathrm{~h}$ and further treated with $20 \mu \mathrm{M}$ GANT61 or vehicle for $48 \mathrm{~h}$ before subjected to mitoSOX red flow cytometric analysis. Bar graph represents the increase in the mean fluorescence intensity of mitoSOX red-positive cells measured in each experimental condition. Values are the average of independent measurements (mean $\pm \mathrm{SEM} ; n=3$ ). ${ }^{* * *}, p<0.001$, compared to GANT61-treated cells.

shown in Figure 7A, ethidium bromide treatment resulted in a marked reduction of PCR products for COX1, D-loop and ND6. There was no difference in the levels of GAPDH PCR product between LO68 and LO68 $\rho^{0}$ cells, indicating that nuclear DNA was not depleted in the process of establishing LO68 $\rho^{0}$ cells (Figure 7A). Next, LO68 and LO68 $\rho^{0}$ cells were treated with GANT61 $(10-20 \mu \mathrm{M})$ for $48 \mathrm{~h}$ before staining with mitoSOX red. In LO68 cells, exposure to GANT61 for $48 \mathrm{~h}$ resulted in an induction of mitochondrial superoxide formation. MitoSOX red oxidation was significantly reduced in LO68 $\rho^{0}$ cells, indicating that a functional respiratory chain is required for the GANT61 induction of superoxide formation (Figure 7B). To determine whether functional mitochondrial respiratory chain is important for mediating GANT61-induced apoptosis, we compared apoptosis in LO68 and LO68 $\rho^{0}$ cells exposed to GANT61 (10 and $20 \mu \mathrm{M})$ for $48 \mathrm{~h}$. As shown in Figure 7C, GANT61 increased apoptosis in a dosedependent manner in LO68 cells as assessed by annexin V staining. In contrast, GANT61 induced negligible apoptosis in LO68 $\rho^{0}$ cells after exposure to GANT61. Furthermore, to exclude the possibility that LO68 $\rho^{0}$ cells were resistant to apoptosis because of their loss of a functional mitochondrial respiratory chain, LO68 and LO68 $\rho^{0}$ cells were treated with cisplatin (10 and $20 \mu \mathrm{M})$, a standard chemotherapeutic drug known to induce apoptosis in LO68 cells, for $48 \mathrm{~h}$, and apoptosis was assessed by annexin $\mathrm{V}$ staining. As shown in Figure 7D, LO68 and LO68 $\rho^{0}$ cells undergo apoptosis when treated with cisplatin. Also there was no statistical difference in the level of apoptosis between LO68 and LO68 $\rho^{0}$ cells as demonstrated by annexin V assay $(p>0.05)$. Taken together, these results suggest that GANT61-induced apoptosis is mediated by mitochondrial superoxide.

\section{DISCUSSION}

Targeting the Hh pathway either through RNAinterference knockdown of GLI1 and GLI2 or using Gli inhibitors, has been shown to induce growth inhibition and cell death in mesothelioma cells and xenograft tumors in vivo $[13,21,22]$. A promising anticancer agent GANT61, with Gli inhibitory activity, displayed potent cytotoxic activity against diverse human cancer types including MMe [13-15, 23-25]. On the basis of computational docking and surface plasmon resonance data, GANT61 has been proposed to mediate its pro-apoptotic effect by binding directly to GLI1, which in turn inhibits GLI1 from binding to DNA $[9,26]$. There is evidence that GANT61 appears to have a novel anticancer mechanism that differs from other Hh antagonists. Recent studies have reported 
A

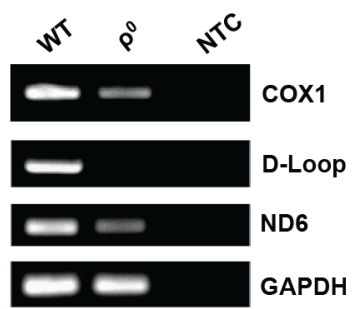

C

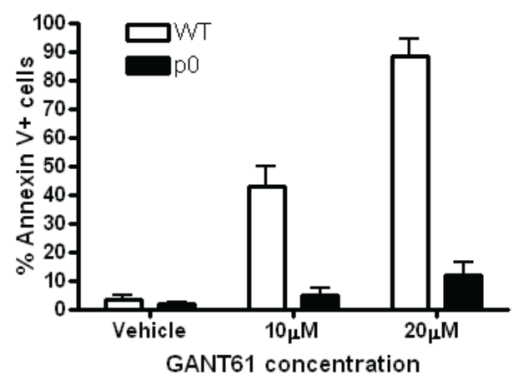

B

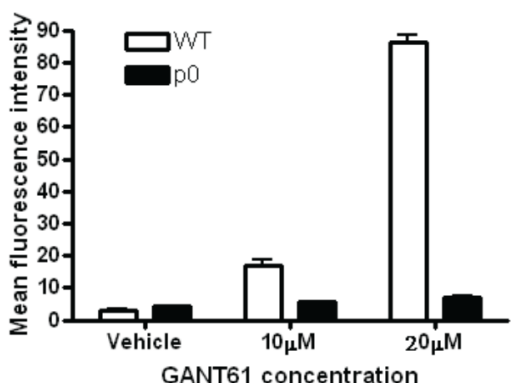

D

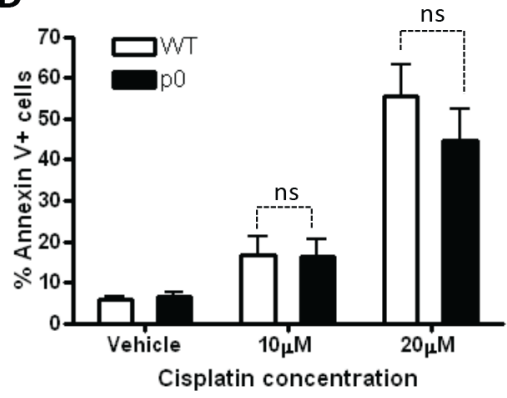

Figure 7: Mitochondrial ROS is required for GANT61-mediated apoptosis. (A) Depletion of mitochondrial DNA in LO68 cells. Total DNA (20 ng) from wild-type (WT) and mitochondrial DNA-depleted $\rho^{0}$ LO68 cells were subjected to PCR amplification using primers that were designed from specific regions of the mitochondrial DNA coding for COX1, D-loop and ND6. GAPDH was included as a control for nuclear DNA-encoded gene. NTC, no template control. (B) Detection of mitochondrial superoxide on GANT61 treatment. WT or $\rho^{0}$ LO68 cells were treated with 10-20 $\mu$ M GANT61 or vehicle for $48 \mathrm{~h}$. The cells were then stained with mitoSOX red, and the fluorescence was measured by flow cytometry. Bar graph represents the increase in the mean fluorescence intensity of mitoSOX red-positive cells measured in each experimental condition. Values are the average of independent measurements (mean \pm SEM; $n=3$ ). (C) $\rho^{0}$ LO68 cells are resistant to GANT61-induced apoptosis. WT or $\rho^{0}$ LO68 cells were treated with 10-20 $\mu$ M GANT61 for 48 h. Apoptosis was then measured by annexin-V/7AAD assay. Data represent the mean \pm SEM of three independent experiments. (D) No apparent difference was observed in the sensitivity to apoptosis by cisplatin in WT or $\rho^{0}$ LO68 cells. Cells were treated with 10-20 $\mu \mathrm{M}$ Cisplatin for $48 \mathrm{~h}$. Apoptosis was then measured by annexin-V/7AAD assay. Data represent the mean \pm SEM of three independent experiments. ns, not significantly different from untreated control cells.

that GANT61 triggers apoptosis via induction of DNA double strand breaks and activation of ATM-Chk2 DNA damage response in colon cancer cells [10,27].

Using a panel of MMe cell lines, we have demonstrated that GANT61-induced anti-proliferative effects were related to the inhibition of cell growth, as confirmed by reduction of cell growth and induction of G1 cell cycle arrest. Our data also show that GANT61 induced apoptotic cell death in LO68 cells in a concentrationand time-dependent manner. We present data indicating that GANT61 specifically acts on the Hh-Gli pathway, as demonstrated by a reduction in the expression levels of downstream pathway effectors GLI1 and GLI2 and Gli target genes PTCH1 and Bcl-2. GANT61 also significantly decreased the Gli-luciferase reporter activity in a dose-dependent manner. These results are consistent with previous reports in HEK294 cells transiently overexpressing GLI1 and colon cancer cells with constitutively active Hh signaling $[9,15]$. At this juncture, our data corroborate previous findings that GANT61 inhibits Hh signaling at the level of Gli transcription factors $[9,14,15]$. However, silencing GLI1 and GLI2, individually or together, were not sufficient to induce cell death in LO68 cells, strongly suggesting that GANT61induced apoptosis was not associated with Gli inhibition. Our data is in contrast to previous siRNA experiments, where silencing of GLI1 resulted in increased apoptosis and reduced level of anti-apoptotic Bcl-2 in HCC, glioma and breast cancer cells [28-30]. Thus, it is possible that GANT61-induced apoptosis may be initiated by another factor other than Gli inhibition.

An alternative explanation for Gli-independent induction of apoptosis by GANT61 is production of ROS. Previous reports have shown that certain chemotherapeutic drugs can induce caspase-independent apoptosis that is brought about by the production of ROS [31, 32]. The present studies showed that ROS were generated concomitantly with apoptosis in a dose- and timedependent manner in LO68 cells upon treatment of cells with GANT61. Interestingly, similar to GANT61-induced apoptosis, Gli silencing by siRNA showed that although GLI1 and GLI2 were downregulated in LO68 cells, they did not appear to be involved in induction of ROS generation. Furthermore, the apoptogenic role of ROS production was 


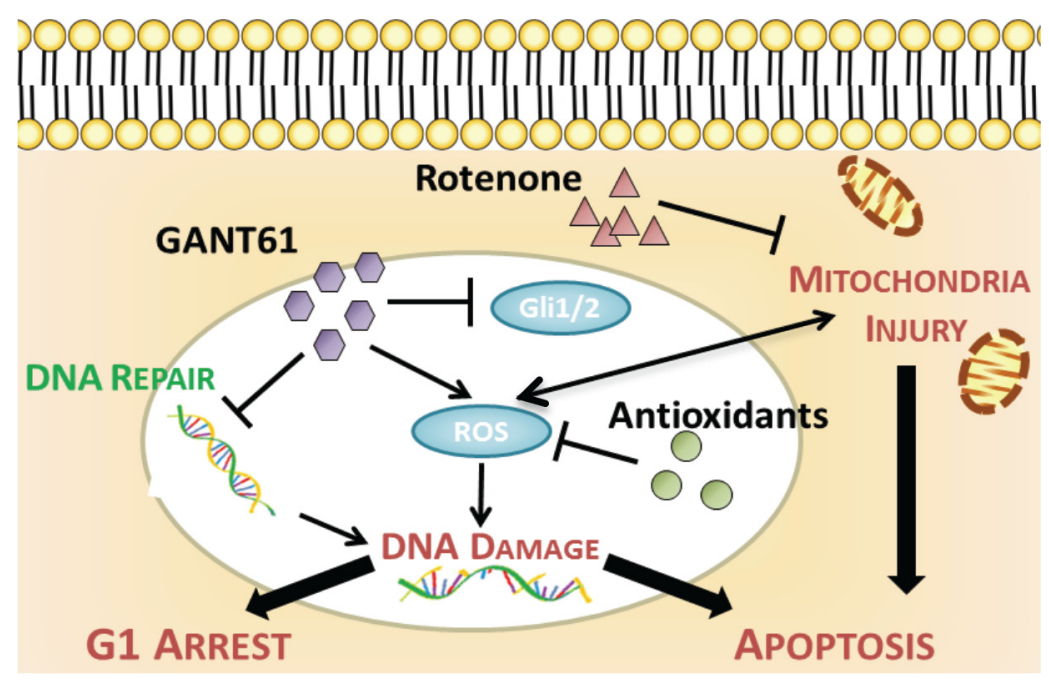

Figure 8: Schematic representation of the proposed mechanism of GANT61-induced apoptosis in MMe cells. GANT61 triggers the production of mitochondrial ROS independent of Hh/Gli signaling. GANT61-induced ROS causes DNA damage and reduced DNA repair, which leads to G1 cell cycle arrest and apoptosis. Pretreatment of cells with either antioxidants or rotenone can reverse this ROS-induced apoptosis.

supported by the ability of two general antioxidants, NAC and GSH, to rescue cells from GANT61-induced apoptosis. However, it is hard to determine the specific ROS species that are generated from GANT61 exposure in LO68 cells. It seems likely that the superoxide produced in the mitochondria might play an important role in the induction of apoptosis. Approximately 1-2\% of electrons can "leak" to oxygen to form superoxide in a reaction mediated mainly by complex I and III of the mitochondrial respiratory chain [33]. To identify the source of ROS, rotenone, a complex I inhibitor, was able to reduce GANT61-induced ROS production and rescue LO68 cells from GANT61-induced apoptosis when it was added to cells prior to GANT61 exposure. This result implied that GANT61-induced ROS might come from mitochondria. Because the blockade of GANT61-induced apoptosis by rotenone was partial, there could also be other sites of ROS production. Another major site of ROS generation is plasma membrane NADPH oxidase [34].

Mitochondrial DNA-depleted $\rho^{\circ}$ cells lack a working respiratory chain and are not able to generate ATP and ROS within mitochondria $[35,36]$. We thus hypothesized that the depletion of mitochondrial DNA in LO68 $\rho^{\circ}$ cells may interfere with ROS production and in turn apoptosis, after treatment with GANT61. Consistent with our hypothesis, LO68 $\rho^{\circ}$ cells showed resistance to GANT61, and lower mitochondrial superoxide levels were also observed after GANT61 treatment. The resistance of LO68 $\rho^{\circ}$ cells to GANT61 was not associated with a decreased susceptibility to apoptosis, as indicated by the equal sensitivity to cisplatin of wild-type and $\rho^{\circ}$ cells. Overall, our results are consistent with the hypothesis that susceptibility to GANT61 stems from exaggerated production of ROS from the mitochondrial respiratory chain.
In conclusion, the present study not only demonstrates the therapeutic potential of GANT61 in MMe, but also demonstrates a novel mechanism for GANT61-induced G1 phase arrest and apoptosis in MMe cells. This is the first report of apoptosis induced by GANT61 via generation of mitochondrial ROS. Based on the findings in this paper, we propose a model showing the relationship of GANT61, ROS and mitochondria in the induction of growth suppression and apoptosis of MMe cells (Figure 8). Our results offer an initial proof-of-concept that mitochondrial ROS-mediated anticancer mechanisms may be exploited for therapeutic benefits in MMe.

\section{MATERIAL AND METHODS}

\section{Cell lines and culture conditions}

The cell lines MSTO-211H, NCI-H28, HCT116 and HT29 were obtained from the American Type Culture Collection; JU77, LO68, NO36, ONE58 and STY51 were gifts from Professor Bruce W. Robinson [37]; GAY2911, OLD1612 and VGE were derived from pleural fluids or tumors of MMe patients. Cells were cultured in Dulbecco's modified Eagle's medium supplemented with $10 \%$ fetal bovine serum (Serana), $4 \mathrm{mM}$ L-glutamine (Life Technologies), 100 units/ml penicillin (Life Technologies) and $100 \mu \mathrm{g} / \mathrm{ml}$ streptomycin (Life Technologies) in a humidified $37^{\circ} \mathrm{C}$ incubator with $5 \% \mathrm{CO}_{2}$.

\section{TaqMan quantitative real-time PCR analysis (qRT-PCR)}

qRT-PCR was performed as described previously [38]. mRNA expression of genes was quantified using the following TaqMan gene expression arrays (Applied 
Biosystems): GLI1 (hs01110766_m1), GLI2 (hs01119974 m1), PTCH1 (hs00181117_m1) and PGK1 (4326318E1006008). The levels of each gene were normalized to $P G K 1$ mRNA and presented as fold change with respect to untreated cells for each gene.

\section{Cell proliferation assay}

Cell proliferation was determined using the methylene blue assay as previously described [39]. Briefly, cells were seeded in 96-well plates and treated the following day with GANT61 $(0.2-50 \mu \mathrm{M})$ (Tocris Bioscience) or dimethyl sulfoxide (DMSO) (Sigma Aldrich) as vehicle control. Following treatment, cells were fixed with $4 \%$ paraformaldehyde (Sigma Aldrich) for $10 \mathrm{~min}$ at $4^{\circ} \mathrm{C}$ followed by staining with $2 \%$ methylene blue/0.01M borate $(\mathrm{pH} 8.5)$ solution (Sigma Aldrich). Excess dye was then washed off using $0.01 \mathrm{M}$ borate buffer $(\mathrm{pH}$ 8.5) and the methylene blue dye from cells were extracted with $1: 1(\mathrm{v} / \mathrm{v})$ ethanol and $0 . \mathrm{I} \mathrm{M}$ hydrochloric acid and quantitated at $650 \mathrm{~nm}$ on a Wallac 1420 VICTOR2 multilabel plate reader (Perkin Elmer). Half maximal inhibitory concentrations $\left(\mathrm{IC}_{50}\right)$ were determined using Graphpad Prism 4.03 software (Graphpad Software, Inc.).

\section{Cell cycle analysis by DNA content}

Cells were treated with $20 \mu \mathrm{M}$ GANT61 for 24-72 $\mathrm{h}$ then fixed with $70 \%$ ethanol at $4{ }^{\circ} \mathrm{C}$. Cells were stained with $50 \mu \mathrm{g} / \mathrm{ml}$ propidium iodide (Sigma Aldrich) in the presence of $100 \mu \mathrm{g} / \mathrm{ml}$ DNase-free RNase A (Life Technologies) for $30 \mathrm{~min}$ at room temperature. Stained cells were analyzed for DNA content using a FACSCalibur flow cytometer (BD Biosciences) and quantified using the FlowJo software (Tree Star, Inc.).

\section{Detection of apoptosis}

Detection of apoptotic cells was performed with the PE Annexin V Apoptosis Detection kit (BD Biosciences) according to the manufacturer's protocol. Briefly, cells were harvested after drug treatment, washed twice with ice-cold phosphate buffered saline (PBS) and incubated with Annexin V PE conjugate and 7-aminoactinomycin $\mathrm{D}$ (7AAD) for $15 \mathrm{~min}$ in the dark. Stained cells were analyzed by flow cytometry and quantified using the FlowJo software.

\section{ROS detection}

Intracellular ROS production was determined by loading cells with $20 \mu \mathrm{M}$ 6-carboxy-2',7'dichlorodihydrofluorescein diacetate ( $\left.\mathrm{CH}_{2} \mathrm{DCFDA}\right)$ (Life Technologies) at $37^{\circ} \mathrm{C}$ for $45 \mathrm{~min}$. Intramitochondrial superoxide production was determined by loading cells with $5 \mu \mathrm{M}$ mitoSOX red mitochondrial superoxide indicator (Life Technologies) at $37^{\circ} \mathrm{C}$ for $45 \mathrm{~min}$. Red and green fluorescence emissions were analyzed by flow cytometry using excitation/emission wavelengths of $488 / 530 \mathrm{~nm}$ and $488 / 585 \mathrm{~nm}$ for $\mathrm{CH}_{2}$ DCFDA and mitoSOX red, respectively.

\section{RNA interference}

Cells were grown to $80 \%$ confluence and transfected with 100 nM GLI1 siRNA (Santa Cruz Biotechnology), GLI2 siRNA (Sigma Aldrich) or negative control siRNA (Santa Cruz Biotechnology) using Lipofectamine 2000 transfection reagent (Life Technologies) according to the manufacturer's instructions.

\section{Gli luciferase reporter assay}

Gli transcriptional activity was measured using a Cignal Gli Reporter (luc) kit (SABiosciences) according to manufacturer's instructions. Briefly, cells were seeded in 12-well plates $24 \mathrm{~h}$ before transfection. Cells were cotransfected with 500 ng of Gli luciferase reporter construct and a Renilla luciferase construct (40:1 ratio) using Lipofectamine 2000 transfection reagent, with a 9:1 ratio (v/w) of Lipofectamine 2000 to DNA. Cells were harvested using the Dual-Glo Luciferase assay system (Promega) $48 \mathrm{~h}$ after transfection according to the manufacturer's instruction. Luciferase activity was measured using a microplate reader. All reporter assays were normalized to Renilla luciferase activity.

\section{Western blot analysis}

Cells were harvested, lysed using CelLytic M mammalian cell lysis/extraction reagent (Sigma Aldrich), and the protein concentrations determined by NanoDrop 2000c Spectrophotometer (Thermo Scientific). Proteins (30 $\mu \mathrm{g})$ were separated by sodium dodecyl sulfatepolyacrylamide gel electrophoresis and electrotransferred onto nitrocellulose membrane (Millipore), which was then blocked with $5 \%$ nonfat milk or bovine serum albumin (Sigma Aldrich) in $0.1 \%$ Tris-buffered saline-Tween 20 for $1 \mathrm{~h}$ at room temperature. The membranes were incubated at $4^{\circ} \mathrm{C}$ overnight with the indicated primary antibodies. Antibodies recognizing GLI1, GLI2 and Bcl-2 were purchased from Cell Signaling Technology. As a protein loading control, membranes were probed with a mouse anti- $\beta$-actin monoclonal antibody (Santa Cruz Biotechnology). After repeated washing to remove unbound antibodies, the membranes were further incubated with horseradish peroxide-conjugated anti-rabbit or antimouse secondary antibodies (Cell Signaling Technology) for $1 \mathrm{~h}$ at room temperature. Chemiluminescent detection of antibody binding was performed using the Immobilon Western HRP Substrate (Millipore). 


\section{Generation of $\mathrm{LO68} \mathrm{q}^{0}$ cells}

LO68 $\rho^{0}$ cells were generated by treating LO68 cells with $100 \mathrm{ng} / \mathrm{ml}$ ethidium bromide (Sigma Aldrich), $50 \mu \mathrm{g} /$ $\mathrm{ml}$ uridine (Sigma Aldrich) and $1 \mathrm{mM}$ sodium pyruvate (Life Technologies).

\section{PCR amplification}

Total DNA was isolated from cells using the PureLink Genomic DNA kit (Life Technologies), according to the manufacturer's instructions. PCR primers that amplify genes coding for cytochrome c oxidase subunit 1 (COX1), D-loop, NADH dehydrogenase 6 (ND6) and glyceraldehyde-3-phosphate dehydrogenase (GADPH) were obtained from published literature [40, 41]. PCR amplification was performed as described [41] and the PCR products were visualized on $1.5 \%$ agarose gels.

\section{Statistical analysis}

Statistical calculations were performed using Graphpad Prism 5 software (Graphpad Software, Inc.). Student's $t$-test was used to determine statistical differences between two groups. Statistical differences between multiple groups were calculated using oneway ANOVA analysis and Tukey's multiple comparison post-hoc test. $P<0.05$ was considered statistically significant.

\section{ACKNOWLEDGEMENTS}

The authors wish to thank Winthrop Professor Bruce Robinson for MMe cells; Associate Professor Matthew Linden and Ms. Tracey Lee-Pullen (Centre for Microscopy, Characterisation and Analysis, University of Western Australia) for support with flow cytometry; Ms. Hui Min Cheah for help with illustration. This study was supported by grants from the National Health and Medical Research Council, Australia (Project ID number 572676) and the Mesothelioma Applied Research Foundation, USA (to SEM). CBL was supported by the University of Western Australia International Postgraduate Research Scholarship and Lung Institute of Western Australia PhD Top Up Scholarship.

\section{REFERENCES}

1. Robinson B, Lake R. Advances in malignant mesothelioma. N Engl J Med. 2005; 353:1591-1603.

2. Ceresoli GL, Gridelli C, Santoro A. Multidisciplinary treatment of malignant pleural mesothelioma. Oncologist. 2007; 12:850-863.

3. Ingham $\mathrm{P}$ McMahon $\mathrm{A}$. Hedgehog signaling in animal development: paradigms and principles. Genes Dev. 2001; 15:3059-3087.
4. van den Brink GR. Hedgehog signaling in development and homeostasis of the gastrointestinal tract. Physiological reviews. 2007; 87:1343-1375.

5. Stone D, Hynes M, Armanini M, Swanson T, Gu Q, Johnson R, Scott M, Pennica D, Goddard A, Phillips H, Noll M, Hooper J, de Sauvage F, Rosenthal A. The tumoursuppressor gene patched encodes a candidate receptor for Sonic hedgehog. Nature. 1996; 384:129-134.

6. Taipale J, Cooper MK, Maiti T, Beachy PA. Patched acts catalytically to suppress the activity of Smoothened. Nature. 2002; 418:892-897.

7. Katoh Y, Katoh M. Hedgehog target genes: mechanisms of carcinogenesis induced by aberrant hedgehog signaling activation. Curr Mol Med. 2009; 9:873-886.

8. Axelson M, Liu K, Jiang $\mathrm{X}$, He K, Wang J, Zhao H, Kufrin D, Palmby T, Dong Z, Russell AM, Miksinski S, Keegan P. Pazdur R. U.S. Food and Drug Administration approval: vismodegib for recurrent, locally advanced, or metastatic basal cell carcinoma. Clin Cancer Res. 2013; 19:2289-2293.

9. Lauth M, Bergstrom A, Shimokawa T, Toftgard R. Inhibition of GLI-mediated transcription and tumor cell growth by small-molecule antagonists. Proc Natl Acad Sci U S A. 2007; 104:8455-8460.

10. Mazumdar T, Devecchio J, Agyeman A, Shi T, Houghton JA. Blocking Hedgehog survival signaling at the level of the GLI genes induces DNA damage and extensive cell death in human colon carcinoma cells. Cancer Res. 2011; 71:5904-5914.

11. Kawabata N, Ijiri K, Ishidou Y, Yamamoto T, Nagao H, Nagano S, Maeda S, Komiya S, Setoguchi T. Pharmacological inhibition of the Hedgehog pathway prevents human rhabdomyosarcoma cell growth. International journal of oncology. 2011; 39:899-906.

12. Desch P, Asslaber D, Kern D, Schnidar H, Mangelberger D, Alinger B, Stoecher M, Hofbauer SW, Neureiter D, Tinhofer I, Aberger F, Hartmann TN, Greil R. Inhibition of GLI, but not Smoothened, induces apoptosis in chronic lymphocytic leukemia cells. Oncogene. 2010; 29:4885-4895.

13. You M, Varona-Santos J, Singh S, Robbins DJ, Savaraj N, Nguyen DM. Targeting of the Hedgehog signal transduction pathway suppresses survival of malignant pleural mesothelioma cells in vitro. The Journal of thoracic and cardiovascular surgery. 2013; 147:508-516.

14. Wickstrom M, Dyberg C, Shimokawa T, Milosevic J, Baryawno N, Fuskevag OM, Larsson R, Kogner P, Zaphiropoulos PG, Johnsen JI. Targeting the hedgehog signal transduction pathway at the level of GLI inhibits neuroblastoma cell growth in vitro and in vivo. International journal of cancer Journal international du cancer. 2013; 132:1516-1524.

15. Mazumdar T, DeVecchio J, Shi T, Jones J, Agyeman A, Houghton JA. Hedgehog signaling drives cellular survival in human colon carcinoma cells. Cancer Res. 2011; 71:1092-1102. 
16. Wang Y, Han C, Lu L, Magliato S, Wu T. Hedgehog signaling pathway regulates autophagy in human hepatocellular carcinoma cells. Hepatology. 2013; 58:995-1010.

17. Fang J, Seki T, Maeda H. Therapeutic strategies by modulating oxygen stress in cancer and inflammation. Advanced drug delivery reviews. 2009; 61:290-302.

18. Sena LA, Chandel NS. Physiological roles of mitochondrial reactive oxygen species. Molecular cell. 2012; 48:158-167.

19. Wondrak GT. Redox-directed cancer therapeutics: molecular mechanisms and opportunities. Antioxidants \& redox signaling. 2009; 11:3013-3069.

20. Bigelow RL, Chari NS, Unden AB, Spurgers KB, Lee S, Roop DR, Toftgard R, McDonnell TJ. Transcriptional regulation of bcl-2 mediated by the sonic hedgehog signaling pathway through gli-1. J Biol Chem. 2004; 279:1197-1205.

21. Li H, Lui N, Cheng T, Tseng HH, Yue D, Giroux-Leprieur E, Do HT, Sheng Q, Jin JQ, Luh TW, Jablons DM, He B. Gli as a novel therapeutic target in malignant pleural mesothelioma. PLoS One. 2013; 8:e57346.

22. Bosco-Clement G, Zhang F, Chen Z, Zhou HM, Li H, Mikami I, Hirata T, Yagui-Beltran A, Lui N, Do HT, Cheng T, Tseng HH, Choi H, Fang LT, Kim IJ, Yue D, et al. Targeting Gli transcription activation by small molecule suppresses tumor growth. Oncogene. 2014; 33:2087-2097.

23. Tostar U, Toftgard R, Zaphiropoulos PG, Shimokawa T. Reduction of human embryonal rhabdomyosarcoma tumor growth by inhibition of the hedgehog signaling pathway. Genes \& cancer. 2010; 1:941-951.

24. Pan D, Li Y, Li Z, Wang Y, Wang P, Liang Y. Gli inhibitor GANT61 causes apoptosis in myeloid leukemia cells and acts in synergy with rapamycin. Leukemia research. 2012; 36:742-748.

25. Nagao H, Ijiri K, Hirotsu M, Ishidou Y, Yamamoto T, Nagano S, Takizawa T, Nakashima K, Komiya S, Setoguchi T. Role of GLI2 in the growth of human osteosarcoma. The Journal of pathology. 2011; 224:169-179.

26. Agyeman A, Jha BK, Mazumdar T, Houghton JA. Mode and specificity of binding of the small molecule GANT61 to GLI determines inhibition of GLI-DNA binding. Oncotarget. 2014; 5:4492-4503.

27. Agyeman A, Mazumdar T, Houghton JA. Regulation of DNA damage following termination of Hedgehog $(\mathrm{HH})$ survival signaling at the level of the GLI genes in human colon cancer. Oncotarget. 2012; 3:854-868.

28. Thomas ZI, Gibson W, Sexton JZ, Aird KM, Ingram SM, Aldrich A, Lyerly HK, Devi GR, Williams KP. Targeting GLI1 expression in human inflammatory breast cancer cells enhances apoptosis and attenuates migration. Br J Cancer. 2011; 104:1575-1586.
29. Wang K, Pan L, Che X, Cui D, Li C. Gli1 inhibition induces cell-cycle arrest and enhanced apoptosis in brain glioma cell lines. Journal of neuro-oncology. 2010; 98:319-327.

30. Chen XL, Cao LQ, She MR, Wang Q, Huang XH, Fu XH. Gli-1 siRNA induced apoptosis in Huh7 cells. World journal of gastroenterology : WJG. 2008; 14:582-589.

31. Lambert C, Apel K, Biesalski HK, Frank J. 2-methoxyestradiol induces caspase-independent, mitochondria-centered apoptosis in DS-sarcoma cells. International journal of cancer Journal international du cancer. 2004; 108:493-501.

32. Ruefli AA, Ausserlechner MJ, Bernhard D, Sutton VR, Tainton KM, Kofler R, Smyth MJ, Johnstone RW. The histone deacetylase inhibitor and chemotherapeutic agent suberoylanilide hydroxamic acid (SAHA) induces a cell-death pathway characterized by cleavage of Bid and production of reactive oxygen species. Proc Natl Acad Sci U S A. 2001; 98:10833-10838.

33. Orrenius S, Gogvadze V, Zhivotovsky B. Mitochondrial oxidative stress: implications for cell death. Annual review of pharmacology and toxicology. 2007; 47:143-183.

34. Jiang F, Zhang Y, Dusting GJ. NADPH oxidase-mediated redox signaling: roles in cellular stress response, stress tolerance, and tissue repair. Pharmacological reviews. 2011; 63:218-242.

35. Chandel NS, Schumacker PT. Cells depleted of mitochondrial DNA (rho0) yield insight into physiological mechanisms. FEBS letters. 1999; 454:173-176.

36. King MP, Attardi G. Human cells lacking mtDNA: repopulation with exogenous mitochondria by complementation. Science. 1989; 246:500-503.

37. Manning LS, Whitaker D, Murch AR, Garlepp MJ, Davis MR, Musk AW, Robinson BW. Establishment and characterization of five human malignant mesothelioma cell lines derived from pleural effusions. International journal of cancer Journal international du cancer. 1991; 47:285-290.

38. Lim CB, Prele CM, Cheah HM, Cheng YY, Klebe S, Reid G, Watkins DN, Baltic S, Thompson PJ, Mutsaers SE. Mutational analysis of hedgehog signaling pathway genes in human malignant mesothelioma. PLoS One. 2013; 8:e66685.

39. Oliver MH, Harrison NK, Bishop JE, Cole PJ, Laurent GJ. A rapid and convenient assay for counting cells cultured in microwell plates: application for assessment of growth factors. Journal of cell science. 1989; 92:513-518.

40. Wu K, Jiang SW, Couch FJ. p53 mediates repression of the BRCA2 promoter and down-regulation of BRCA2 mRNA and protein levels in response to DNA damage. J Biol Chem. 2003; 278:15652-15660.

41. Moro L, Arbini AA, Yao JL, di Sant'Agnese PA, Marra E, Greco M. Mitochondrial DNA depletion in prostate epithelial cells promotes anoikis resistance and invasion through activation of PI3K/Akt2. Cell death and differentiation. 2009; 16:571-583. 